\title{
Supply kits for antenatal and childbirth care: a systematic review
}

\author{
Alicia Aleman ${ }^{1 *}$ (D), Giselle Tomasso ${ }^{1}$, María Luisa Cafferata ${ }^{1}$, Mercedes Colomar ${ }^{1}$ and Ana Pilar Betran ${ }^{2}$
}

\begin{abstract}
Introduction: It is critical to increase the uptake of interventions proven to be effective to improve maternal and perinatal outcomes. Supply kits have been suggested to be a feasible strategy designed to ensure timely availability and effective follow-up of care.
\end{abstract}

Objective: We conducted a systematic review to summarize the evidence on the uptake, effectiveness and safety of supply kits for maternal care.

Search strategy: MEDLINE, the Cochrane Pregnancy and Childbirth Group's Trials Register, Campbell Collaboration, Lilacs, Embase and unpublished studies were searched.

Selection criteria: Studies that reported the efficacy, safety and use of supply kits for maternal healthcare were eligible. Participants were pregnant women or in childbirth. Supply kits were defined as a collection of medicines, supplies or instruments packaged together with the aim of conducting a healthcare task.

Data collection and analysis: Two reviewers independently performed the screening, data extraction, and methodological and quality assessment.

Main results: 24 studies were included: 4 of them were systematic reviews and 20 primary studies. Eighteen studies evaluated a so-called "clean delivery kit". In all but two studies, the kits were used by more than half of the participants. A meta-analysis was deemed inappropriate due to the heterogeneity in study design, in the components of the interventions implemented, in the content of the kits, and in outcomes. Nine studies assessed neonatal outcomes and found statistically significant reductions in cord infection, sepsis and tetanus-related mortality in the intervention group. Three studies showed evidence of reduced neonatal mortality (OR 0.52, 0.60 and 0.71 ) with statistically significant confidence intervals in all cases. Four studies reported odd ratios for maternal mortality, but only one showed evidence of a statistically significant decrease in this outcome but it was ascribed to hand washing prior to childbirth and not with the use of kits.

Conclusion: This review suggests potential benefits in the use of supply kits to improve maternal and neonatal health. However, the observational nature of the studies, the heterogeneity and the use of kits incorporated within complex interventions limit the interpretation of the findings.

Keywords: Supply kits, Clean delivery kits, Pregnancy, Childbirth, Maternal mortality, Neonatal mortality

\footnotetext{
* Correspondence: aaleman@unicem-web.org

${ }^{1}$ Montevideo Clinical and Research Unit, Avda Italia s/n, Hospital de Clinical,

11600 Montevideo, Uruguay

Full list of author information is available at the end of the article
} 


\section{Plain english summary}

Supply kits are considered a potential strategy to improve maternal and child health, as they provide medication or diagnostic tests at the same time. This review searched for all published studies that tested the use of supply kits and assessed their effectiveness and safety. The studies found were of moderate to low quality. Most of them suggested benefits for mothers and babies, namely, reduced mortality and morbidity, with the use of supply kits. We concluded that although more research is needed to more comprehensively evaluate this strategy, it seems potentially useful for maternal and child health.

\section{Background}

Improving maternal health and reducing child mortality were two of the eight Millennium Development Goals (MDGs) adopted by the international community in 2000. Between 1990 and 2015, the number of global maternal deaths dropped by $43 \%$ (from 532,000 to 303,000 ) [1]. To accelerate this decline, countries have established the Sustainable Development Goals (SDGs) as a follow-up initiative. Two targets are included under SDG 3: to reduce the global maternal mortality ratio to less than 70 per 100,000 births, with no country having a maternal mortality rate more than twice the global average, and to end preventable deaths of newborns, with all countries aiming to reduce neonatal mortality to 12 per 1000 live births [2].

Most maternal deaths are preventable, as the healthcare solutions to prevent or manage potential maternal complications are well known [1]. However, several factors can prevent the uptake of interventions that have been proven to be efficient and beneficial, specifically during pregnancy and delivery [3]. Supply chain deficiencies and stock-outs are among the most limiting barriers that hinder the delivery of effective practices in poor-resource settings [4-9]. Supply kits (packaged supplies targeting women, healthcare providers or health facilities) have been proposed to be a simple and lowcost intervention that can address various challenges routinely encountered in low-income countries. In the area of maternal and newborn health, supply kits have been designed to focus on issues ranging from timely availability of effective treatment in emergency situations and avoidance of stock-outs for routine care to achieving clean childbirth and reducing the incidence of infections and the associated complications, particularly in areas where women give birth at home [10-13]. Different types of supply kits have been implemented and tested as single or multicomponent interventions, and reviews assessing their effectiveness have been conducted, the latest of which was published in 2012 [14]. The timely update of the evidence is important for policy makers and implementers planning to use supply kits to improve care in areas where supply change deficiencies and stock-outs are a major bottleneck to reaching women with effective screening and treatment interventions. Even if the proportion of women delivering in health facilities is increasing, births often occur in a place with sub-standard hygienic conditions within the facility, without appropriately trained staff or without the appropriate medicines and conditions $[15,16]$.

The objective of this systematic review was to update and summarise the evidence with focus on the uptake, effectiveness and safety of supply kits for maternal care, particularly for antenatal and childbirth care at both institutional and community level.

\section{Methods}

The methodology and reporting of results in this systematic review followed all steps proposed in the PRISMA statement [17]. This review was registered in the Prospero Centre for Reviews and Dissemination, University of York, with the number CRD42016043145 [18].

\section{Eligibility criteria of studies Type of studies}

Any study that reported the use of supply kits for maternal healthcare was eligible for inclusion, regardless of the study design, sample size, period and setting (e.g., nationwide, facility-based).

\section{Type of participants}

Pregnant women at any period of gestation or during labour and childbirth were eligible, regardless of women's obstetric or medical characteristics, level of risk, education and socio-economic status.

\section{Type of intervention}

Supply kits. Kits were defined as a collection of medicines, supplies or instruments packaged together with the aim of conducting a healthcare task (e.g., antenatal care kit, caesarean section kit, delivery kit). This review included kits designed for individual use (e.g., kits given to each woman for childbirth at home) and kits designed for health facilities that contained supplies for use in their service population (e.g., all supplies necessary to conduct antenatal care for 100 women). Supply boxes of a single product (e.g., ARV) or kits that included only educational interventions were not included in this review.

\section{Type of outcomes}

The primary outcomes were as follows: maternal, perinatal and neonatal mortality; stillbirth (as defined by the authors); use of supply kits, including the proportion of women whose health care included application of the kits; number of ANC visits; low birth weight; complications of pregnancy, including 
prolonged anaemia, obstructed labour, eclampsia, postpartum haemorrhage, and postpartum depression (as defined by the authors); and referral to a health facility for any complication during pregnancy, delivery, or the postpartum period. The secondary outcomes included the following: iron/folate supplementation; tetanus toxoid immunization; institutional delivery; birth attended by a healthcare provider; use of bed nets (to avoid insect bites and prevent malaria); urine exams; syphilis and HIV diagnosis and treatment; initiation of breastfeeding within one hour of birth; wrapping babies within $30 \mathrm{~min}$; and health care seeking for maternal and/or neonatal morbidities.

\section{Search strategy for identification of studies}

The terms included in the search were medical supplies, clean, sanitary, disposable equipment, kit, birth kit, toolkit, package, box, prenatal care, antenatal care, pregnancy complications, pregnancy, postpartum period, labour, obstetric, intrapartum, partum, peripartum, and childbirth. An additional file shows the search terms in more detail [see Additional file 1]. Two experienced librarians in the Institute for Clinical Effectiveness (IECS, Buenos Aires, Argentina) and in the World Health Organization (WHO) assisted with the search. We searched MEDLINE (1966 to 2016), Embase (1980 to 2016) Lilacs (1982 to 2016), the Cochrane Pregnancy and Childbirth Group Trial Register and the Campbell Collaboration. Websites related to grey literature were also searched to identify unpublished studies. No limits regarding publication date or language were applied.

Process of study identification, selection and data extraction Citations identified through the search strategy of the electronic databases were imported into Early Review Organizing Software (EROS), and duplicates were deleted [19]. Four reviewers in pairs independently assessed the studies at each stage. In the first stage, all identified citations imported into EROS were screened based on the title and the abstract to select potentially relevant citations for full-text evaluation. When information in the title/abstract was insufficient to determine the inclusion/exclusion criteria, the full text was retrieved and evaluated. In the second stage, the full text of all the selected citations was retrieved and assessed. Citations fulfilling the inclusion criteria were included. Data were extracted using a structured data extraction form designed specifically for this review by the authors. Discrepancies were resolved through discussion and consensus. When data in the original publication were not sufficiently detailed, the authors were contacted for additional information.

\section{Risk of bias assessment}

Both experimental and observational designs were eligible for inclusion in this review. We assessed the risk of bias of all included studies with quality assessment tools specifically intended for each study design using the currently internationally recommended tools. For experimental studies (randomized trials), we used the tool proposed by the Cochrane Collaboration to assess the quality of these types of studies [20]. The dimensions assessed with this tool are as follows: quality of randomization methods, allocation concealment, blinding of participants and of evaluators, incomplete outcome data and selective reporting of outcomes [20]. For observational studies, the assessment of the risk of bias considered three major criteria (methods for selecting participants, methods for measuring exposure and outcome variables, and methods to control for confounding) and two minor criteria (statistical methods and conflict of interest) [21-23]. For before and after studies, the assessment of the risk of bias considered blindness and reliability of the outcome measure and follow-up of participants [24, 25].

The quality of the systematic reviews was assessed using GRADE [26]. Primary studies included in the systematic reviews were evaluated for inclusion in our review, and those not retrieved in our search were included as appropriate.

\section{Analysis and reporting}

The association between the use of supply kits and maternal, perinatal and neonatal outcomes was assessed using crude and adjusted odds ratios (ORs) and relative risks (RRs) with 95\% confidence intervals or rates, depending on the study design. Meta-analyses were not performed due to the expected differences in the designs and data of the primary studies (different type of data, data collection, populations). The findings were reported considering two dimensions: the time when the supply kits were used (antenatal or during childbirth) and the outcomes related to the use of supply kits (uptake of the kits, maternal morbidity and mortality and neonatal morbidity and mortality).

\section{Findings}

\section{Results of the search}

The search strategy identified 2495 unique citations. After assessing the titles and the abstracts for inclusion criteria, 2299 were excluded, and after a full-text evaluation, 172 additional citations were excluded. Finally, 24 manuscripts were selected, namely, 4 systematic reviews [14, 27-29] and 20 primary studies reporting data on the uptake, effectiveness and safety of the use of supply kits. All four systematic reviews included observational studies and searched for packaged interventions that 
included not only supply kits but also educational and behavioural components. The primary studies of the reviews were checked against our search results. One article [10] of the 20 primary studies included had not been identified through our search strategy and was thus added [See Additional file 2 for characteristic of the reviews]. Figure 1 shows the flowchart of our systematic review.

Table 1 and Table 2 show the characteristics of the 20 studies included in this review. The majority of the studies used a cross-sectional design $(n=12)$, and only one was a randomized controlled trial [30]. Most studies occurred in developing countries in Asia $(n=9)$ and Africa $(n=8)$. Two studies were from Oceania, and one study was conducted in the United Kingdom. The sample size of the studies ranged from 19 to 118,160 participants. In five studies, supply kits were the only component assessed by the authors, whereas 15 studies evaluated a multicomponent intervention that included the use of a clean delivery kit (CDK) together with an educational/behavioural component. All but one described kits designed for individual use either at home or in the health facility in the absence of complications.
One study [11] used an emergency obstetric kit: a single-use code box that provided rapid access to effective treatment for specific emergencies.

In most of the studies, women, birth attendants, health workers or traditional birth attendants (TBAs) received a CDK intended for childbirth either at home or in the health facility (if the woman received the CDK and she delivered at a health facility, she brought the CDK with her to the health facility). Seven studies reported data on interventions that were implemented at home only. In 10 studies, the intervention was either at home or in health facilities, and in three, the intervention was only at the health facility. Nine studies reported neonatal and perinatal outcomes, eight reported maternal outcomes, and ten studies reported data on the uptake of the supply kits (See Table 1).

\section{Kits' effectiveness and safety during pregnancy}

Women were provided supply kits to be used during pregnancy in only one study (HIV-related kits) [12]. In all the other studies, the kits provided were intended for childbirth and were given either directly to the women

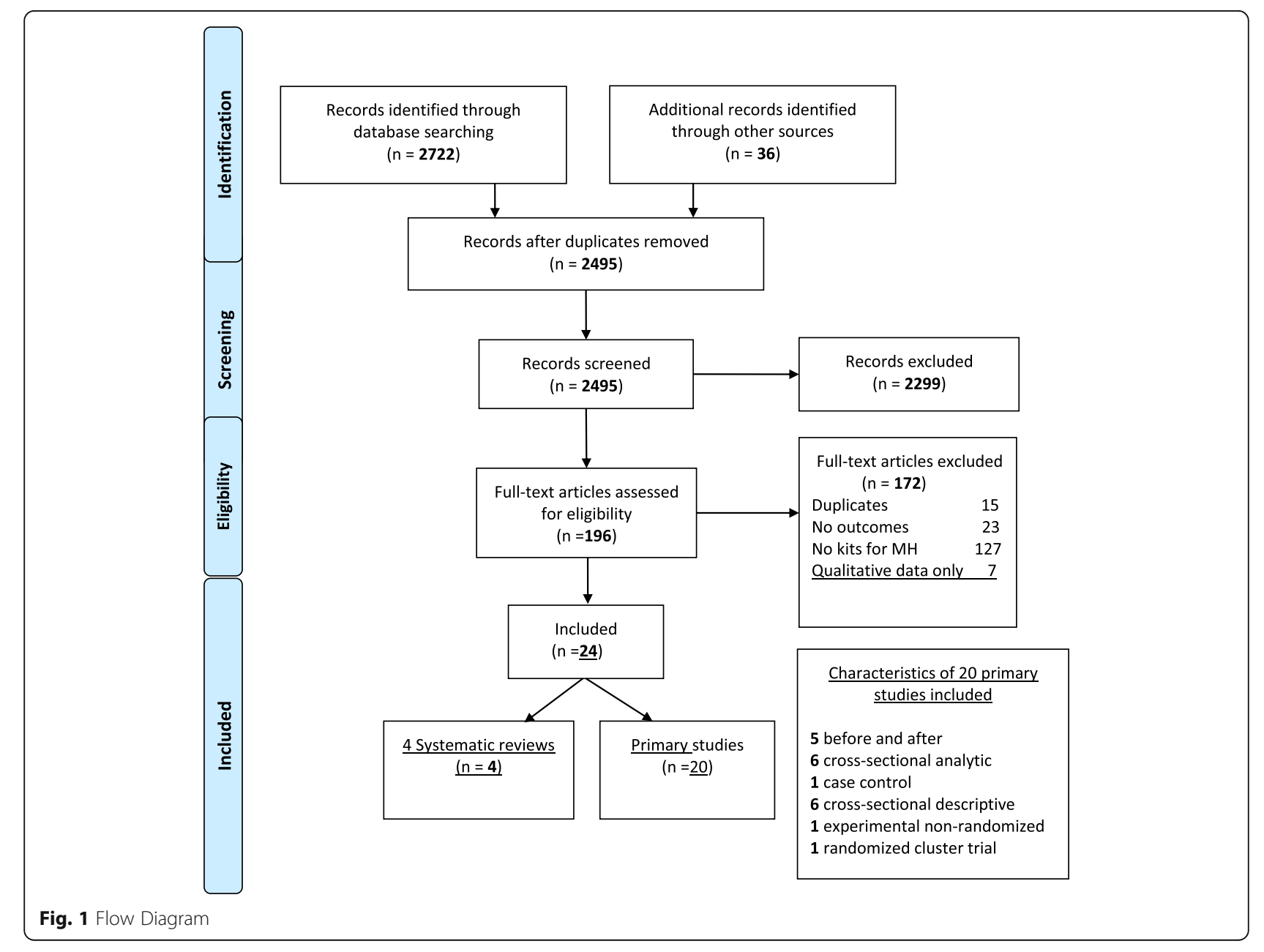


Table 1 General characteristics of 20 Primary included studies

\begin{tabular}{|c|c|}
\hline Characteristic & $N(\%)$ \\
\hline \multicolumn{2}{|l|}{ Type of manuscript } \\
\hline Articles in peer-reviewed journals & $18(90.0)$ \\
\hline Congress Abstracts & $2(10.0)$ \\
\hline \multicolumn{2}{|l|}{ Method } \\
\hline Quantitative only & $19(95.0)$ \\
\hline Both qualitative and quantitative & $1(5.0)$ \\
\hline \multicolumn{2}{|l|}{ Study design } \\
\hline Randomized controlled trial & $1(5.0)$ \\
\hline Experimental non-randomized & $1(5.0)$ \\
\hline Cross-sectional & $12(60.0)$ \\
\hline Case Control & $1(5.0)$ \\
\hline Before-and-after intervention & $5(25.0)$ \\
\hline \multicolumn{2}{|l|}{ Region } \\
\hline Asia & $9(45.0)$ \\
\hline Africa & $8(40.0)$ \\
\hline Europe & $1(5.0)$ \\
\hline Oceania & $2(10.0)$ \\
\hline \multicolumn{2}{|l|}{ Place of Delivery } \\
\hline Health facility based & $3(15.0)$ \\
\hline Home childbirth & $7(35.0)$ \\
\hline Both (Home and Health facility) & $10(50.0)$ \\
\hline \multicolumn{2}{|l|}{ Participants } \\
\hline \multicolumn{2}{|l|}{ Study subjects who received the intervention } \\
\hline Pregnant women & $9(45.0)$ \\
\hline Birth attendants, health workers, TBAs & $8(40.0)$ \\
\hline Women during childbirth & $5(25.0)$ \\
\hline Newborns & $1(5.0)$ \\
\hline \multicolumn{2}{|c|}{ Study subjects in which outcomes were assessed } \\
\hline Pregnant women & $5(25.0)$ \\
\hline Women during delivery & $17(85.0)$ \\
\hline Neonates & $8(40.0)$ \\
\hline Women during postpartum & $5(25.0)$ \\
\hline Infants & $1(5.0)$ \\
\hline \multicolumn{2}{|l|}{ Sample size } \\
\hline$>10,000$ & $5(25.0)$ \\
\hline $10,000-1000$ & $6(30.0)$ \\
\hline$<1000$ & $9(45.0)$ \\
\hline \multicolumn{2}{|l|}{ Type of components } \\
\hline Only the kits & $5(25.0)$ \\
\hline Kits + behavioral intervention & $4(20.0)$ \\
\hline Kits + behavioral + other components & $1(5.0)$ \\
\hline Kits + training & $6(25.9)$ \\
\hline Kits + training + other components & $4(20.0)$ \\
\hline
\end{tabular}

or to the healthcare providers. They were provided either during pregnancy or at the time of birth. McDougal evaluated the effect of a kit of ARV drugs on preventing mother-to-child transmission of HIV in Lesotho (12). The kit, distributed at health centres for individual use, contained all the necessary pregnancy, delivery and early postnatal antiretroviral medications for the mother and the infant. No differences were found between the two groups of women before and after the intervention in terms of the coverage and quality of ANC or infant immunization within three months of birth (a proxy of HIV testing for babies). However, there was a significant reduction in HIV-positive women delivering in health facilities $(57.7 \%$ pre-intervention and $48.9 \%$ post intervention, $p<0.05)$.

\section{Kits' effectiveness and safety during childbirth and the immediate postpartum period}

Nineteen studies evaluated the use of supply kits during childbirth and the immediate postpartum period. These were supply kits that aimed to promote clean delivery, and they focused predominantly on components to achieve the " 6 cleans" proposed by the WHO (clean attendant hands, surface, blade, cord tie, towels to dry then wrap the baby, and cloth to wrap the mother) [31]. Table 3 displays the content of the supply kits in each study. All the supply kits were intended for vaginal delivery, with no studies evaluating supply kits for caesarean section. The supply kits were distributed at no cost in seven (35\%) studies. Out-of-pocket payment was required in one study, while in another study, whether kits were charged for varied according to region. For the remaining 10 studies (50\%), information on charges to the women was unclear.

Ten studies including 53,068 women reported data on the uptake of the use of supply kits [30, 32-40] (See Table 4). The median use of supply kits was $62 \%$, ranging from $15 \%$ to $100 \%$. Studies that reported the uptake of supply kits both at home and at a health facility found that the use of kits for births at home was always higher than the use at health facilities [4, 32]. One study reported a high acceptability and use but did not share the data [41]. Uptake or impact of the supply kit according to the receiver (e.g., health provider or women) was not addressed specifically in any study.

The studies included in this review reported the effect of the intervention as a whole, regardless of the number and type of components (Table 1). We could not separate the effect of the different components, and thus the effect estimates reported hereafter refer to the complete intervention, not exclusively to the supply kits. Only five studies assessed the effect of the supply kits alone. One evaluated an emergency kit [11], one a homeopathic delivery kit [33], and three assessed the effect of CDK 
Table $\mathbf{2}$ Characteristics of 20 primary studies included

\begin{tabular}{|c|c|c|c|c|c|c|c|c|c|}
\hline \multirow[t]{2}{*}{ Author } & \multirow[t]{2}{*}{ Year } & \multirow{2}{*}{$\begin{array}{l}\text { Number of } \\
\text { participants }\end{array}$} & \multirow[t]{2}{*}{ Country } & \multirow{2}{*}{$\begin{array}{l}\text { Home or } \\
\text { facility } \\
\text { based }\end{array}$} & \multirow{2}{*}{$\begin{array}{l}\text { Type of } \\
\text { study/design }\end{array}$} & \multicolumn{2}{|l|}{ Participants } & \multirow{2}{*}{$\begin{array}{l}\text { Components of } \\
\text { the Intervention } \\
\text { (by study subjects) }\end{array}$} & \multirow[t]{2}{*}{ Outcomes } \\
\hline & & & & & & $\begin{array}{l}\text { Study subjects } \\
\text { who received } \\
\text { the intervention }\end{array}$ & $\begin{array}{l}\text { Study subjects } \\
\text { in whom the } \\
\text { outcomes were } \\
\text { measured }\end{array}$ & & \\
\hline $\begin{array}{l}\text { Balsara } \\
\text { et al }\end{array}$ & 2009 & 349 women & Egypt & $\begin{array}{l}\text { Home } 284 \\
\text { Health } \\
\text { facility } 65\end{array}$ & $\begin{array}{l}\text { Cross-sectional } \\
\text { analytical }\end{array}$ & $\begin{array}{l}\text { Health providers } \\
\text { TBAs (Dayas) } \\
\text { Pregnant women }\end{array}$ & $\begin{array}{l}\text { Pregnant } \\
\text { women } \\
\text { Women } \\
\text { during delivery }\end{array}$ & $\begin{array}{l}\text { CDK (Health providers) } \\
\text { CDK + training(TBAs) } \\
\text { CDK+ use instructions } \\
\text { (Pregnant women) }\end{array}$ & $\begin{array}{l}\text { Number of ANC } \\
\text { visits } \\
\text { Use of CDK } \\
\text { Clean delivery } \\
\text { practices } \\
\text { CDK acceptability }\end{array}$ \\
\hline $\begin{array}{l}\text { Calvert } \\
\text { et al }\end{array}$ & 2007 & 19 women & $\begin{array}{l}\text { United } \\
\text { Kingdom }\end{array}$ & $\begin{array}{l}\text { Health } \\
\text { facility }\end{array}$ & $\begin{array}{l}\text { Cross-sectional } \\
\text { study no analytic }\end{array}$ & $\begin{array}{l}\text { Women in labor } \\
\text { and delivery }\end{array}$ & $\begin{array}{l}\text { Women in } \\
\text { labor, delivery } \\
\text { and } \\
\text { postpartum }\end{array}$ & $\begin{array}{l}\text { Homeopathic remedies } \\
\text { kit (Women in labor } \\
\text { and delivery) }\end{array}$ & $\begin{array}{l}\text { Use of kit } \\
\text { Benefit during } \\
\text { birth }\end{array}$ \\
\hline $\begin{array}{l}\text { Darmstadt } \\
\text { et al }\end{array}$ & 2009 & $\begin{array}{l}334 \text { women } \\
6 \text { lost }\end{array}$ & Egypt & $\begin{array}{l}\text { Home } 276 \\
\text { Health } \\
\text { facility } 54\end{array}$ & $\begin{array}{l}\text { Cross-sectional } \\
\text { study no analytic }\end{array}$ & $\begin{array}{l}\text { TBAs (raedat, } \\
\text { dayas, nurses) } \\
\text { Community health } \\
\text { workers } \\
\text { Skilled birth attendants } \\
\text { Pregnant women }\end{array}$ & $\begin{array}{l}\text { Women } \\
\text { during delivery } \\
\text { Newborns }\end{array}$ & $\begin{array}{l}\text { CDK (Health providers) } \\
\text { CDK + training(TBAs) } \\
\text { CDK+ instructions } \\
\text { (Pregnant women) }\end{array}$ & $\begin{array}{l}\text { Cord infection } \\
\text { Puerperal } \\
\text { infection }\end{array}$ \\
\hline $\begin{array}{l}\text { Dickerson } \\
\text { et al }\end{array}$ & 2010 & $\begin{array}{l}980 \text { women } \\
378 \text { outreach } \\
\text { providers }\end{array}$ & Tibet & $\begin{array}{l}\text { Home } 452 \\
\text { Health } \\
\text { facility } \\
495\end{array}$ & $\begin{array}{l}\text { Cross-sectional } \\
\text { study no analytic }\end{array}$ & $\begin{array}{l}\text { Pregnant women } \\
\text { Outreach Health } \\
\text { workers } \\
\text { Laypersons }\end{array}$ & $\begin{array}{l}\text { Women } \\
\text { during delivery }\end{array}$ & $\begin{array}{l}\text { CDK + training (to all) + } \\
\text { antenatal and postnatal } \\
\text { micronutrient } \\
\text { supplementation(women) }\end{array}$ & $\begin{array}{l}\text { Use of CDK } \\
\text { Use of beneficial } \\
\text { practices } \\
\text { Breast-feeding }\end{array}$ \\
\hline $\begin{array}{l}\text { Garner } \\
\text { et al }\end{array}$ & 1994 & $\begin{array}{l}126 \text { women } \\
131 \text { neonates }\end{array}$ & $\begin{array}{l}\text { Papua New } \\
\text { Guinea }\end{array}$ & Home & $\begin{array}{l}\text { Before and } \\
\text { after study }\end{array}$ & Pregnant women & Neonates & $\begin{array}{l}\text { CDK+ use instructions } \\
\text { (women) }\end{array}$ & $\begin{array}{l}\text { Neonatal sepsis } \\
\text { Use of the kits } \\
\text { Fever }\end{array}$ \\
\hline Greenwood & 1990 & $\begin{array}{l}15 \text { villages } \\
\text { with PHC } \\
\text { and non-PHC; } \\
673 \text { women } \\
\text { before the } \\
\text { introduction } \\
\text { of the Program } \\
\text { and } 1913 \text { after }\end{array}$ & Gambia & Home & $\begin{array}{l}\text { Before and } \\
\text { after study }\end{array}$ & TBAs & $\begin{array}{l}\text { Pregnant } \\
\text { women } \\
\text { Women } \\
\text { during delivery } \\
\text { Newborns } \\
\text { Women } \\
\text { during } \\
\text { postpartum } \\
\text { period }\end{array}$ & $\begin{array}{l}\text { Training + obstetric } \\
\text { package }^{\mathrm{a}} \text { (TBA) }\end{array}$ & $\begin{array}{l}\text { Number of ANC } \\
\text { visits Maternal } \\
\text { mortality } \\
\text { Maternal morbidity } \\
\text { Perinatal mortality } \\
\text { Stillbirth } \\
\text { Neonatal death } \\
\text { Tetanus } \\
\text { immunization } \\
\text { Pre-eclampsia }\end{array}$ \\
\hline $\begin{array}{l}\text { Hassan } \\
\text { et al }\end{array}$ & 2012 & $\begin{array}{l}225 \text { women } \\
82 \text { health workers }\end{array}$ & Pakistan & $\begin{array}{l}\text { Home } 100 \\
\text { Health } \\
\text { facility } \\
125\end{array}$ & $\begin{array}{l}\text { Cross-sectional, } \\
\text { questionnaire } \\
\text { study, no analytic }{ }^{c}\end{array}$ & TBA, women. & $\begin{array}{l}\text { Women } \\
\text { during delivery } \\
\text { Health workers }\end{array}$ & CDK & $\begin{array}{l}\text { Use of CDK } \\
\text { Use of clean } \\
\text { delivery practices }\end{array}$ \\
\hline $\begin{array}{l}\text { Jokhio } \\
\text { et al }\end{array}$ & 2005 & $\begin{array}{l}\text { 19,557 women } \\
\text { TBAs }\end{array}$ & Pakistan & Home & $\begin{array}{l}\text { Experimental } \\
\text { randomized } \\
\text { cluster trial }\end{array}$ & $\begin{array}{l}\text { TBAs in seven } \\
\text { talukas (rural } \\
\text { Larkana) }\end{array}$ & $\begin{array}{l}\text { Pregnant } \\
\text { women } \\
\text { Women } \\
\text { during delivery }\end{array}$ & $\begin{array}{l}\text { CDK + training (TBAs) } \\
\text { VS NO training/ } \\
\text { NO CDK }\end{array}$ & $\begin{array}{l}\text { Perinatal mortality } \\
\text { Maternal mortality } \\
\text { hemorrhage } \\
\text { obstructed labor } \\
\text { puerperal sepsis } \\
\text { eclampsia, } \\
\text { abortion } \\
\text { Referral }\end{array}$ \\
\hline $\begin{array}{l}\text { Kapoor } \\
\text { et al }\end{array}$ & 1991 & 7687 deliveries & India & $\begin{array}{l}\text { Home } \\
6652 \\
\text { Health } \\
\text { facility } \\
1035\end{array}$ & $\begin{array}{l}\text { Cross-sectional } \\
\text { study no analytic }\end{array}$ & $\begin{array}{l}\text { TBAs } \\
\text { Pregnant women }\end{array}$ & $\begin{array}{l}\text { Pregnant } \\
\text { women } \\
\text { Women } \\
\text { during delivery } \\
\text { Neonates }\end{array}$ & $\begin{array}{l}\text { Training to conduct } \\
\text { deliveries(TBAs) } \\
\text { CDK + tetanus toxoid } \\
\text { immunization + Training } \\
\text { in Clean delivery } \\
\text { practices (women) }\end{array}$ & $\begin{array}{l}\text { Tetanus toxoid } \\
\text { coverage } \\
\text { Neonatal mortality } \\
\text { Tetanus neonatal } \\
\text { mortality }\end{array}$ \\
\hline $\begin{array}{l}\text { McDougal } \\
\text { et al }\end{array}$ & 2012 & 1545 women & Lesotho & $\begin{array}{l}\text { Health } \\
\text { facility }\end{array}$ & $\begin{array}{l}\text { Before and after } \\
\text { study }\end{array}$ & Pregnant women & $\begin{array}{l}\text { Pregnant } \\
\text { women } \\
\text { Women } \\
\text { during delivery } \\
\text { Women } \\
\text { during } \\
\text { postpatum } \\
\text { Infants }\end{array}$ & $\begin{array}{l}\text { MPP }^{\text {b: pregnancy, }} \\
\text { Intrapartum and } 7 \text { day } \\
\text { post-partum ARVs } \\
\text { (women) + neonatal } \\
\text { ARVs for } 7 \text { or } \\
28 \text { days(infants) }\end{array}$ & $\begin{array}{l}\text { Number of ANC } \\
\text { visits } \\
\text { HIV Positive } \\
\text { women with } 4 \\
\text { antenatal visits } \\
\text { HIV negative } \\
\text { women with } 4 \\
\text { ANC visits Children } \\
\text { with DNA } \\
\text { PCR test at } \\
53 \text { months }\end{array}$ \\
\hline $\begin{array}{l}\text { Meegan } \\
\text { et al }\end{array}$ & 2001 & 118,160 births & $\begin{array}{l}\text { Kenya } \\
\text { Tanzania }\end{array}$ & $\begin{array}{l}\text { Home } \\
\text { Health } \\
\text { facility }\end{array}$ & $\begin{array}{l}\text { Experimental } \\
\text { non-randomized } \\
\text { clinical trial }\end{array}$ & TBAs & Neonates & CDK + training (TBAs) & $\begin{array}{l}\text { Neonatal tetanus } \\
\text { Mortality under } \\
6 \text { weeks }\end{array}$ \\
\hline
\end{tabular}


Table 2 Characteristics of 20 primary studies included (Continued)

\begin{tabular}{|c|c|c|c|c|c|c|c|c|c|}
\hline \multirow[t]{2}{*}{ Author } & \multirow[t]{2}{*}{ Year } & \multirow{2}{*}{$\begin{array}{l}\text { Number of } \\
\text { participants }\end{array}$} & \multirow[t]{2}{*}{ Country } & \multirow{2}{*}{$\begin{array}{l}\text { Home or } \\
\text { facility } \\
\text { based }\end{array}$} & \multirow{2}{*}{$\begin{array}{l}\text { Type of } \\
\text { study/design }\end{array}$} & \multicolumn{2}{|l|}{ Participants } & \multirow{2}{*}{$\begin{array}{l}\text { Components of } \\
\text { the Intervention } \\
\text { (by study subjects) }\end{array}$} & \multirow[t]{2}{*}{ Outcomes } \\
\hline & & & & & & $\begin{array}{l}\text { Study subjects } \\
\text { who received } \\
\text { the intervention }\end{array}$ & $\begin{array}{l}\text { Study subjects } \\
\text { in whom the } \\
\text { outcomes were } \\
\text { measured }\end{array}$ & & \\
\hline $\begin{array}{l}\text { Mukasa } \\
\text { et al }\end{array}$ & 2012 & 3116 women & $\begin{array}{l}\text { Uganda } \\
\text { Tanzania }\end{array}$ & $\begin{array}{l}\text { Home } \\
\text { Health } \\
\text { facility }\end{array}$ & $\begin{array}{l}\text { Cross-sectional } \\
\text { study no } \\
\text { analytic }\end{array}$ & Pregnant women & $\begin{array}{l}\text { Women } \\
\text { during delivery }\end{array}$ & $\begin{array}{l}\text { CDK + misoprostol + } \\
\text { information(women) }\end{array}$ & $\begin{array}{l}\text { Use of CDK } \\
\text { Acceptability }\end{array}$ \\
\hline $\begin{array}{l}\text { Ouma } \\
\text { et al }\end{array}$ & 2012 & $\begin{array}{l}7080 \text { deliveries } \\
\text { before } \\
8269 \text { deliveries } \\
\text { after }\end{array}$ & Kenya & $\begin{array}{l}\text { Health } \\
\text { facility }\end{array}$ & $\begin{array}{l}\text { Before and } \\
\text { after study }\end{array}$ & $\begin{array}{l}\text { Birth attendants } \\
\text { (BA) }\end{array}$ & $\begin{array}{l}\text { Women with } \\
\text { an obstetric } \\
\text { emergency }\end{array}$ & $\begin{array}{l}\text { Obstetric emergency } \\
\text { kit (BA) }\end{array}$ & $\begin{array}{l}\text { Maternal mortality } \\
\text { due to hemorrhage, } \\
\text { pre eclampsia/ } \\
\text { eclampsia, cardio } \\
\text { pulmonary arrest }\end{array}$ \\
\hline $\begin{array}{l}\text { Quaiyum } \\
\text { et al }\end{array}$ & 2012 & $\begin{array}{l}118,594 \\
\text { women }\end{array}$ & Bangladesh & Home & $\begin{array}{l}\text { Before and } \\
\text { after study }\end{array}$ & $\begin{array}{l}\text { TBAs } \\
\text { Pregnant women } \\
\text { from selected } \\
\text { upazilas }\end{array}$ & $\begin{array}{l}\text { Women at } \\
\text { delivery }\end{array}$ & $\begin{array}{l}\text { CDK }+ \text { misoprostol }+ \\
\text { blood collection mat + } \\
\text { training (TBAs } \\
\text { and women) }\end{array}$ & Maternal mortality \\
\hline $\begin{array}{l}\text { Raza } \\
\text { et al }\end{array}$ & 2013 & $\begin{array}{l}420 \\
\text { neonates }\end{array}$ & Pakistan & $\begin{array}{l}\text { Home } \\
311 \\
\text { Health } \\
\text { facility } \\
109\end{array}$ & $\begin{array}{l}\text { Matched Case- } \\
\text { control study }\end{array}$ & $\begin{array}{l}\text { Cases: Newborns } \\
\text { with tetanus } \\
\text { Controls: Newborns } \\
\text { without tetanus }\end{array}$ & No applicable & CDK & $\begin{array}{l}\text { Use of CDK } \\
\text { Use of hygienic } \\
\text { practices }\end{array}$ \\
\hline $\begin{array}{l}\text { Seward } \\
\text { et al }\end{array}$ & 2015 & $\begin{array}{l}40,602 \\
\text { deliveries }\end{array}$ & $\begin{array}{l}\text { Nepal } \\
\text { India } \\
\text { Bangladesh }\end{array}$ & Home & $\begin{array}{l}\text { Cross-sectional } \\
\text { analytical }\end{array}$ & $\begin{array}{l}\text { Women during } \\
\text { delivery }\end{array}$ & $\begin{array}{l}\text { Women } \\
\text { during } \\
\text { delivery }\end{array}$ & $\begin{array}{l}\text { CDK training on clean } \\
\text { delivery practices (hand } \\
\text { washing) }\end{array}$ & $\begin{array}{l}\text { Use of CDK } \\
\text { Maternal mortality }\end{array}$ \\
\hline $\begin{array}{l}\text { Seward } \\
\text { et al }\end{array}$ & 2012 & $\begin{array}{l}19,754 \\
\text { deliveries }\end{array}$ & $\begin{array}{l}\text { Nepal } \\
\text { India } \\
\text { Bangladesh }\end{array}$ & Home & $\begin{array}{l}\text { Cross-sectional } \\
\text { analytical }\end{array}$ & $\begin{array}{l}\text { Women during } \\
\text { delivery }\end{array}$ & Neonates & $\begin{array}{l}\text { CDK training on clean } \\
\text { delivery practices }\end{array}$ & Neonatal mortality \\
\hline Tsu & 2000 & $\begin{array}{l}1600 \\
\text { women }\end{array}$ & Nepal & Home & $\begin{array}{l}\text { Cross-sectional } \\
\text { analytical }\end{array}$ & $\begin{array}{l}\text { Women who } \\
\text { delivered a } \\
\text { live newborn }\end{array}$ & Newborn & CDK & $\begin{array}{l}\text { Cord infection } \\
\text { Newborn } \\
\text { infection } \\
\text { Maternal infection }\end{array}$ \\
\hline $\begin{array}{l}\text { Vallely } \\
\text { et al }\end{array}$ & 2016 & $\begin{array}{l}200 \\
\text { women }\end{array}$ & $\begin{array}{l}\text { Papua New } \\
\text { Guinea }\end{array}$ & $\begin{array}{l}\text { Home } \\
108 \\
\text { Health } \\
\text { facility } 92\end{array}$ & $\begin{array}{l}\text { Cross-sectional } \\
\text { analytical }\end{array}$ & Pregnant women & $\begin{array}{l}\text { Women } \\
\text { during } \\
\text { delivery } \\
\text { Women } \\
\text { during } \\
\text { postpartum }\end{array}$ & $\begin{array}{l}\text { CDK + training + } \\
\text { misoprostol (women) }\end{array}$ & $\begin{array}{l}\text { Use of CDK } \\
\text { CDK acceptability }\end{array}$ \\
\hline $\begin{array}{l}\text { Winani } \\
\text { et al }\end{array}$ & 2007 & $\begin{array}{l}3262 \\
\text { women }\end{array}$ & Tanzania & $\begin{array}{l}\text { Home } \\
1792 \\
\text { Health } \\
\text { facility } \\
1186\end{array}$ & $\begin{array}{l}\text { Cross-sectional } \\
\text { analytical }\end{array}$ & Pregnant women & $\begin{array}{l}\text { Women } \\
\text { during } \\
\text { delivery } \\
\text { Women } \\
\text { during } \\
\text { postpartum } \\
\text { Newborns }\end{array}$ & $\begin{array}{l}\text { CDK + (women's } \\
\text { education) }\end{array}$ & $\begin{array}{l}\text { Use of CDK } \\
\text { Cord Infection } \\
\text { Puerperal sepsis }\end{array}$ \\
\hline
\end{tabular}

abstetric package: clean dressing, scissors and string, oral ergometrine, disinfectant, color-coded spring balance for weighing newborns

${ }^{b}$ MPP: Minimum Prevention Mother to Child Transmission Package

${ }^{c}$ Mothers and health workers were asked details about their last delivery

$[35,37,42]$. The latter three evaluated different outcomes, but in all cases, they reported an increase in the use of clean delivery practices with the use of the kits.

Nine studies reported data on the effect of CDK on neonatal outcomes $[10,13,30,35,40,42-45]$ (See Table 5). The only randomized controlled trial in the review reported statistically significant reductions in perinatal and neonatal mortality in the arm that used kits in the context of a complex intervention. Of the other eight observational studies, three showed a protective effect regarding cord infection in the kit group compared to the control group. A protective effect was also reported for tetanus-specific mortality, neonatal sepsis and neonatal mortality. However, in addition to the observational nature of the study, it tested a complex intervention, and thus it was not possible to attribute the reductions in mortality to the use of the supply kit [30].

Eight studies reported data on the effect of kits on maternal outcomes $[10,11,13,30,38-40,46]$ (See Table 6). They were all community-based interventions except for the study by Ouma et al. [11], which was a study on an emergency kit for use in facilities. Three studies $[13,30,40]$ reported lower puerperal infection in the intervention group, although only two were statistically significant, including the randomized controlled trial (which tested a complex intervention and thus multiple components, not only kits) [30]. Postpartum haemorrhage was also lower in the intervention group in three studies [11, 30, 39]. Maternal mortality was lower in the intervention group in three of the five studies that measured this outcome $[11,30,46]$, but the difference 
Table 3 Components of the Kits

\begin{tabular}{|c|c|c|c|c|c|c|c|c|c|c|}
\hline & Soap & Gloves & $\begin{array}{l}\text { Clean plastic } \\
\text { drape }\end{array}$ & $\begin{array}{l}\text { Sterile } \\
\text { razor }\end{array}$ & $\begin{array}{l}\text { Cord tie/ } \\
\text { clamp }\end{array}$ & $\begin{array}{l}\text { Gauze/ } \\
\text { cotton }\end{array}$ & Antiseptic & $\begin{array}{l}\text { Newborn } \\
\text { cap }\end{array}$ & Other & Cost of the kit \\
\hline \multicolumn{11}{|l|}{ Pregnancy } \\
\hline Mc Dougal 2012 & & & & & & & & & HIV treatment & unknown \\
\hline \multicolumn{11}{|l|}{ Childbirth } \\
\hline \multicolumn{11}{|l|}{ Clean Delivery Kits } \\
\hline Balsara 2009 & $x$ & & $x$ & $x$ & $x$ & & & & & free \\
\hline Calvert 2007 & & & & & & & & & $\begin{array}{l}\text { homeopathic } \\
\text { remedies }\end{array}$ & unclear \\
\hline Dickerson 2010 & $x$ & $x$ & $x$ & $x$ & $x$ & $x$ & & $x$ & $\begin{array}{l}\text { vitamins- } \\
\text { micronutrients }\end{array}$ & probably \\
\hline Darmstadt 2009 & & $x$ & $x$ & $x$ & $x$ & $x$ & $x$ & & & free \\
\hline Garner 1994 & & & & $x$ & $x$ & & $x$ & & & free \\
\hline Hassan 2012 & $x$ & $x$ & $x$ & $x$ & $x$ & $x$ & $x$ & $x$ & & unclear \\
\hline Meegan 2001 & & & & $x$ & $x$ & & $x$ & & & free \\
\hline Jokhio 2005 & $x$ & $x$ & & $x$ & $x$ & $x$ & $x$ & & & free \\
\hline Kapoor 1991 & & $x$ & $x$ & $x$ & & & & & & unclear \\
\hline Mukasa 2012 & $x$ & $x$ & $x$ & $x$ & $x$ & $x$ & & & $\begin{array}{l}\text { misoprostol-blood } \\
\text { collection mat }\end{array}$ & unclear \\
\hline Quaiyum 2012 & & & & & & & & & misoprostol & unclear \\
\hline Raza 2013 & $x$ & $x$ & $x$ & $x$ & $x$ & & & & & unclear \\
\hline Seward 2012 & $x$ & & $x$ & $x$ & $x$ & $x$ & & & & $\begin{array}{l}\text { free/very } \\
\text { low cost }\end{array}$ \\
\hline Seward 2015 & $x$ & & $x$ & $x$ & $x$ & $x$ & & & & free/low cost \\
\hline Tsu 2000 & $x$ & & $x$ & $x$ & $x$ & & & & & $\begin{array}{l}\text { free or cost } \\
\text { depending } \\
\text { on region }\end{array}$ \\
\hline Vallely 2016 & $x$ & $x$ & $x$ & $x$ & $x$ & & & & misoprostol & free \\
\hline Winani 2007 & $x$ & & $x$ & $x$ & $x$ & & & & & free \\
\hline \multicolumn{11}{|l|}{ Emergency kits } \\
\hline Ouma 2012 & & & & & & & & & & not applicable \\
\hline \multicolumn{11}{|l|}{ Obstetric care kit } \\
\hline Greenwood 1990 & & & & & & & & & $\begin{array}{l}\text { Scissors, string, } \\
\text { dresses, oral } \\
\text { ergometrine, } \\
\text { balance }\end{array}$ & unclear \\
\hline
\end{tabular}

${ }^{a}$ Components of the clean delivery kit are not described

was not statistically significant in any of them. One of these five studies [38] also tested the effect of hand washing separately from the supply kits. Hand washing prior to delivery independently reduced maternal mortality (OR 0.51 IC 95\% 0.28-0.93).

Ouma et al. implemented an obstetric emergency kit (called E-kit) for the treatment of postpartum haemorrhage, pre-eclampsia/eclampsia and cardiopulmonary arrest in health facilities using a before-and-after design [11]. They reported 27 maternal deaths in the year preceding the introduction of the E-kits and 19 in the first year of the E-kit implementation (among users and nonusers of kits). In the second year of the E-kit implementation, deaths from haemorrhage decreased by $31.6 \%$ overall and there were no maternal deaths in women treated with kits.

Regarding safety, none of the studies reported outcomes that could represent a harm to women or neonates in relation to the use of kits.

\section{Risk of bias}

The quality assessment of each included study is presented in Additional file 3. A summary of the methodological quality assessment of risk of bias is presented for each domain (conflict of interest, control of confounders, methods and 
Table 4 Frequency of use of clean delivery kits

\begin{tabular}{|c|c|c|c|}
\hline & $\begin{array}{l}\text { Global } \\
\text { n/N (\%) }\end{array}$ & $\begin{array}{l}\text { Home } \\
\text { n/N (\%) }\end{array}$ & $\begin{array}{l}\text { Health facility } \\
\mathrm{n} / \mathrm{N}(\%)\end{array}$ \\
\hline Balsara 2009 & 248/349 (71.0) & $214 / 284(75.4)$ & $44 / 65(67.7)$ \\
\hline \multicolumn{4}{|l|}{ Calvert 2007} \\
\hline Before labour & $12 / 19(63.1)$ & & \\
\hline During labour & 15/19 (78.9) & & \\
\hline Pospartum & 19/19 (100.0) & & \\
\hline Dickerson 2010 & 932/962 (96.9) & & \\
\hline \multicolumn{4}{|l|}{ Garner 1994} \\
\hline Razor pack & 22/33 (66.7) & & \\
\hline Clamp pack & $17 / 34(50.0)$ & & \\
\hline Hassan 2012 & $72 / 225(32.0)^{a}$ & & \\
\hline Jokhio 2005 & $8172 / 10114(80.0)$ & & \\
\hline \multicolumn{4}{|l|}{ Raza 2013} \\
\hline Cases & 24/123 (17.1) & & \\
\hline Controls & $99 / 280(35.4)$ & & \\
\hline Seward 2015 & $5210 / 34660(15.05)$ & & \\
\hline Vallely 2016 & $115 / 200(57.5)$ & 99/106 (93.4) & 16/94 (17.0) \\
\hline Winani 2007 & 1820/3058 (59.5) & & \\
\hline
\end{tabular}

aased on women's report

outcomes measure, and selection of participants) and design [See Additional file 4].

Two experimental studies were included in the review [30, 45], although only one was randomized [30]. Randomization, allocation concealment, blinding of assessment, incomplete outcome data and selective reporting showed low risk of bias. There was no blinding of participants in $80 \%$ of the quasi-experimental studies [10-12, 35, 45], and $80 \%$ had low risk of bias regarding the evaluation and measure of the primary outcome. Regarding the follow-up of participants and providers, there was a low risk of bias in $50 \%$ and $75 \%$ of the included studies, respectively. Observational studies with and without a comparison group [13, 32-34, 36, 38-44, 46] had a low risk of bias regarding the selection of participants (82\%) and conflict of interest of authors (91\%). The methods and measurement of the outcomes (information and detection bias) had a low risk of bias in 55\% of studies as well as control of confounders. In summary, almost all studies included in this review were observational, and half of them had moderate to high risk of bias in three main dimensions: methods, measurement of outcomes and adjustment for confounders. Thus, the body of evidence collected was weak in terms of quality.

\section{Discussion}

This systematic review identified 24 manuscripts, of which four were systematic reviews and 20 primary studies (primary data collection or secondary data
Table 5 Neonatal outcomes

\begin{tabular}{|c|c|c|c|}
\hline & $\begin{array}{l}\text { Intervention } \\
\text { group }\end{array}$ & $\begin{array}{l}\text { Control } \\
\text { group }\end{array}$ & $\begin{array}{l}\text { OR adjusted } \\
\text { IC } 95 \%\end{array}$ \\
\hline \multicolumn{4}{|l|}{ Cord infection } \\
\hline Darmstadt 2009 & $14 / 235$ & $13 / 93$ & $0.42(0.18-0.97)$ \\
\hline Tsu 2000 & - & - & $0.45(0.25-0.81)$ \\
\hline Winani $2007^{\mathrm{a}}$ & $3 / 1820$ & $48 / 1238$ & $0.04(0.01-0.12)$ \\
\hline \multicolumn{4}{|l|}{ Sepsis } \\
\hline Garner $1994^{a}$ & $1 / 67$ & $8 / 64$ & $0.11(0.01-0.87)$ \\
\hline Seward 2012 & - & - & $0.28(0.12-0.65)$ \\
\hline \multicolumn{4}{|c|}{ Tetanus-specific mortality ${ }^{b}$} \\
\hline Kapoor 1991 & 0 & 14.6 & - \\
\hline Meegan $2001^{c}$ & 0.75 & 82 & - \\
\hline \multicolumn{4}{|c|}{ All-cause neonatal mortality } \\
\hline Kapoor $1991^{\text {b }}$ & 19.9 & 39.2 & - \\
\hline Seward 2012 & - & - & $0.51(0.35-0.76)$ \\
\hline Jokhio 2005 & 37 & 53 & $0.71(0.62-0.83)$ \\
\hline Greenwood $1990^{a}$ & $54 / 1159$ & $47 / 675$ & $0.65(0.44-0.98)$ \\
\hline \multicolumn{4}{|l|}{ Stillbirth } \\
\hline Greenwood $^{\mathrm{a}}$ & $61 / 1220$ & $37 / 712$ & $0.96(0.63-1.46)$ \\
\hline \multicolumn{4}{|l|}{ Perinatal mortality } \\
\hline Jokhio $2005^{b}$ & 85 & 120 & $0.70(0.59-0.82)$ \\
\hline Greenwood $1990^{a}$ & $99 / 1220$ & $63 / 712$ & $0.91(0.65-1.27)$ \\
\hline
\end{tabular}

anadjusted

ber 1000 livebirths

cbefore and after study

analyses) presenting information on the uptake and impact of supply kits during pregnancy, childbirth or immediate postpartum period. The majority involved single-use kits for clean childbirth and infection prevention. Most studies were published in peer-reviewed journals, had a sample size of less than 1000 women and were observational studies. Only one study was a randomized controlled trial.

In most of the studies, the kits were used by more than half of the participants, with higher use shown at home than at the facility. In general, the group using the supply kits showed better measured outcomes. However, most of the included studies assessed the use of kits in the context of a complex intervention, and thus it was impossible to conclude that the kits were responsible for the observed differences. The groups using supply kits showed a statistically significant positive effect on neonatal outcomes; including reduced cord infection $[13,40,42]$, sepsis [35, 43], neonatal mortality [10, 30, 43] and perinatal mortality [30]. Several studies showed a statistically significant decrease in maternal adverse outcomes in the group using supply kits (see Table 6) including reduction in puerperal infection $[13,30,40]$ and postpartum haemorrhage $[11,30,39]$ and a non- 
Table 6 Maternal outcomes

\begin{tabular}{|c|c|c|c|}
\hline & Kits group & Control group & $\begin{array}{l}\text { OR adjusted } \\
\text { IC } 95 \%\end{array}$ \\
\hline \multicolumn{4}{|l|}{ Puerperal infection } \\
\hline Jokhio 2005 & 78/10093 & $400 / 9432$ & $0.18(0.14-0.22)$ \\
\hline Darmstadt 2009 & $1 / 235$ & $4 / 93$ & $0.11(0.01-1.06)$ \\
\hline Winani 2007 & 19/1798 & $50 / 1380$ & $0.28(0.17-0.48)$ \\
\hline \multicolumn{4}{|c|}{ Post partum hemorrhage } \\
\hline Vallely 2016 & $15 / 112$ & $33 / 88$ & $0.25(0.13-0.52)^{a}$ \\
\hline Jokhio 2005+ & $174 / 10093$ & $259 / 9432$ & $0.62(0.51-0.75)^{\mathrm{a}}$ \\
\hline Ouma $2012^{b}$ & 6/19 (31.6\%) & 14/27 (51.9\%) & \\
\hline \multicolumn{4}{|l|}{ Eclampsia } \\
\hline Jokhio $2005^{d}$ & 23/10093 & 29/9432 & $0.74(0.42-1.28)^{a}$ \\
\hline \multicolumn{4}{|l|}{ Maternal mortality } \\
\hline Seward 2015 & - & - & $\begin{array}{l}1.26(0.62-2.56) / \\
0.51(0.28-0.93)^{\mathrm{e}}\end{array}$ \\
\hline Ouma 2012 & $19 / 8120$ & 27/6935 & $0.60(0.33-1.08)$ \\
\hline Quaiyum $2012^{c}$ & 137 & 338 & - \\
\hline Jokhio 2005 & - & - & $0.74(0.45-1.23)$ \\
\hline Greenwood 1990 & $13 / 1236$ & $7 / 727$ & $1.09(0.94-2.93)^{\mathrm{a}}$ \\
\hline
\end{tabular}

anadjusted OR

${ }^{\mathrm{b}}$ The denominators are maternal deaths

cper 100,000 livebirths

${ }^{d}$ These outcomes were more related to the training component than the CDK use

eUse of kits with all components did not show difference with no kits use. The effect of hand washing prior delivery did show a significant reduction in the odd of maternal death

significant reduction in maternal mortality [11, 30, 46].Meta-analysis was deemed inappropriate due to the heterogeneity in the study designs, in the content of the supply kits, in the definition of compliance in use of supply kits and in the outcomes measured.

One study depicted potential adverse effects related to supply kits: a decrease in institutional childbirth in HIVpositive women who received the kit containing antiretroviral medication. Nonetheless, HIV-positive women compose a very specific group of women and might have different behaviours related to seeking antenatal and delivery care compared to the others [12].

This review showed that the most tested type of supply kit was the CDK. Eighteen studies evaluated kits with all or some of the components of the CDK (Table 2) and showed positive results in increasing clean delivery practices (Table 4). It has been estimated that clean childbirth practices could avert $6-9 \%$ of the 1.16 million newborn deaths in sub-Saharan African countries [31]. However, controversy remains regarding the independent effect of each component or clean practice. Seward et al. [38] conducted the only study that separately examined the effect of kit use and hand washing. They found that while hand washing with soap before delivery was independently associated to a reduction in maternal mortality, kit use had no significant effect on the prevention of maternal deaths. Similarly, Tsu et al. [42] reported that hand washing with soap before cutting the cord vs. not washing hands reduced cord infection even more than using a complete CDK. Balsara et al. [32] found no difference between kits' users and non-users with regard to whether the birth occurred on a clean or unclean surface. These findings suggest that not all CDK components are used or have the same value in improving outcomes. Cultural beliefs may play a role in the understanding of the benefits of using certain components. For example, it could be difficult to understand the usefulness of laying down a plastic sheet during childbirth for women accustomed to delivering in a vertical position [41]. In addition, in our review, only five studies evaluated the effect of supply kits alone, and these studies were all heterogeneous (See Table 1).

Compliance with the "use of CDK" is not defined in the same way across studies, and this adds uncertainty to the conclusions. For example, Winani et al. [40] considered three different scenarios to define when a woman had "used a kit": 1) if she used the full CDK or at least the plastic sheet and either the razor blade or cord tie (or both); 2) if she used the razor blade and cord tie only; or 3) if she used the razor blade alone. This resulted in heterogeneity and weakened the conclusions. In addition, the content of supply kits somewhat varied (see Table 3). However, considering the objective and concept of the kits, the components most frequently included were the sterile razor ( $n=15 \mathrm{kits})$, cord tie ( $n=14$ kits) and soap ( $n=11$ kits).

In this review, all studies except one were conducted in developing countries, mainly in Africa and Asia. In low-resource settings, the use of supply kits is intended to facilitate the provision of interventions by providing all the resources needed for a given situation at one time. In addition, it optimizes the scarce contact between women and the healthcare system in areas where barriers related to accessibility, knowledge and lack of satisfaction prevent women from engaging with the system. In countries with a high prevalence of home births, CDK may be an effective option for reducing newborn infections as well as puerperal sepsis or other genital tract infections following childbirth. The successful implementation of supply kits minimizes the burden of having to separately acquire its contents, which would require knowledge of what is needed and how to use it. This is likely an unrealistic expectation in these settings. The challenges mentioned above justified the implementation of these studies in settings where maternal and neonatal mortality remains high.

We found only one study that described the use of a kit designed to address stock-outs and deficiencies in procurement at the facility level [11]. This kit aimed to 
address the delay in accessing treatment for obstetric emergencies at the health facility level once women were in the facility by considering the context of a dysfunctional supply chain and busy maternity units. In countries with weak and overstretched health systems, stock-outs in medicines and equipment occur routinely and hinder the delivery of effective practices even when women reach the health facility. These kits, which are not intended for individual use, may contain all the supplies required to attend 100 births or to conduct 100 ANC visits, for example. This type of kit has been tested recently in Mozambique in a WHO cluster randomized controlled trial [47]. Further research on the effectiveness and feasibility of this type of kit is warranted because procurement inefficiencies are a chronic and contemporary challenge in many low-resource settings and remote areas.

Even when the acceptability is high and when kits include instructions, the distribution of kits do not guarantee its use. Different studies have employed several approaches to provide access to supply kits including distribution through health facilities, community health workers, TBAs and private providers, such as pharmacists. However, the provider-specific impact on the uptake of supply kits has not been studied. Seward et al. [38] discussed the effect of a complex intervention using a participatory learning and action cycle with women's groups in India and Nepal [38, 48, 49]. The discussions of clean delivery and care-seeking behaviours in women's groups showed an increased uptake in reporting the use of kits in the intervention arm, compared to the control arm.

Available qualitative data is aligned with our results [50]. The main barriers to the implementation of supply kits are those related to socio-cultural and popular beliefs that birth preparation could bring bad luck. Financial constraints and a limited understanding of the instructions on how to use the supply kits have been also identified as accessibility barriers. On the other hand, convenience, the perception of the components as hygienic, and avoidance of delays in receiving care were viewed as satisfactory features that would incentivize the use of the supply kits.

Although four reviews have been previously published on this topic, this systematic review includes an additional four years of evidence, which combined with the current available qualitative evidence [50], it adds a more comprehensive approach to pre-existing knowledge. When the evidence on effectiveness is substandard and based on observational studies, the use of other types and sources of information can be critical to gain a better understanding of the intervention and the pathways of action. This is even more important for complex interventions. In addition, previous reviews have focused on the use of supply kits to reduce neonatal mortality and morbidity [28, 29], kits to conduct family and community interventions [27] and kits designed exclusively for birth [14]. Our review included supply kits for pregnancy and a larger range of maternal and neonatal outcomes.

\section{Strength and limitations}

This review has several strengths. We developed a broad search strategy that included manuscripts and documents not published in peer-reviewed journals. We included studies with a variety of methodological approaches. As the studies originated from different settings in developing countries, we believe that the results of this review can be generalized to low-income settings. This review, however, has some limitations. Importantly, the studies retrieved were mostly observational or had weak experimental designs, which provide a low level of evidence compared to randomized controlled trials. Moreover, the supply kits in these studies were implemented in the context of complex interventions of varying degrees, and it was thus impossible to identify the "active ingredient/s" of the overall intervention. The outcomes were measured in different ways and units, making it difficult to arrive to reliable conclusions. The heterogeneity in the design of the supply kits and its components as well as the definition of compliance of the "use of kit" may hinder the comparability. Lastly, data collection was not always of high quality, and biases may have been present. Most of the studies included in this review were conducted in Africa and Asia, and their results may not be generalizable to other regions such as Latin America.

\section{Conclusions}

Studies found in this review reported a reduction in maternal and neonatal morbidity (particularly infectionrelated morbidity) and neonatal mortality in the groups using supply kits. However, these findings should be interpreted with caution because virtually all the evidence was derived from observational studies and was thus prone to bias and because the effects observed cannot be ascribed to the supply kits alone, given their inclusion in multicomponent interventions. The potential bias of the studies analysed and the heterogeneity hinder the reliability of identifying an overall effect of the kits. It would appear appropriate to continue its use and to expand to other preventive or therapeutic interventions with the inclusion of strong monitoring and evaluation strategies that include comparisons to control groups, as these methods could provide more evidence regarding the real effect of the kits.

\section{Implications for research}

Sub-standard pre-conceptional and ANC care makes it difficult to diagnose and treat conditions occurring 
before or during pregnancy that can affect newborns or place women at increased risk of severe morbidity or mortality. These conditions include syphilis, hepatitis B, HIV, Group B streptococcus, malaria, and even hypertensive disorders of pregnancy, anaemia and urinary infections. A diagnostic and therapeutic package could increase accessibility and compliance. However, these types of kits have not been fully assessed, and research is needed to evaluate their potential effectiveness.

We found one study that implemented emergency kits. Life-threatening conditions during pregnancy and delivery could be effectively diagnosed and treated with specifically designed kits that ensure immediate emergency care. Further research is warranted in this area. Similarly, supply kits for caesarean sections have not been assessed. Finally, although supply kits designed for birth seem to be an effective strategy to improve maternal and neonatal health, there are still several questions that need answering. How and when supply kits should be distributed, who should receive them and what types of promotion and training strategies should be developed for their uptake remain unknown.

\section{Implications for practice}

Positive effects were reported in many of the included studies. Although the evidence comes from observational studies, the use of supply kits could be an appealing feasible strategy for facilitating clean birth practices and access to certain commodities in low-resource settings. Implementation of this strategy requires low-complexity resources and could have a large impact, making supply kits an attractive alternative to increase the quality of care during pregnancy, delivery and the neonatal period, particularly at the community level in low-income countries. Nevertheless, even though the desirable effects seemed to outweigh the adverse effects, close surveillance of these kits should be considered because based on the low quality of evidence found in this review of studies, the authors cannot conclude without a doubt that supply kits are effective in reducing morbidity and mortality.

\section{Additional files}

Additional file 1: Annex I. Search terms for search strategy. (DOCX 15 kb)

Additional file 2: Annex II. Primary studies included in Systematic Reviews retrieved by search strategy. (DOCX $14 \mathrm{~kb}$ )

Additional file 3: Annex III- Quality assessment by individual study. (DOCX $54 \mathrm{~kb}$ )

Additional file 4: Annex IV. A summary of methodological quality assessment of risk of bias by study design. (DOCX $192 \mathrm{~kb}$ )

\section{Acknowledgements}

The authors thank Daniel Comande and Agustin Ciapponi for their help on the development of the search strategy and obtaining the full text of the primary studies. We want to thank to Tomas Allen from World Health Organization, who provided comments on the search strategy.

\section{Funding}

This review was funded by the Reproductive Health Department of the World Health Organization.

\section{Availability of data and materials}

The datasets during and/or analysed during the current study available from the corresponding author on reasonable request.

\section{Authors' contributions}

APB had the original idea. AA, MC, GT and MLC carried out the data extraction, analysis and interpretation of data. AA drafted the article and carried out the statistical analysis. AA, MC, GT, MLC and APB carried out the interpretation of the data and revised the article critically for intellectual content. All authors approved the final draft for publication.

Ethics approval and consent to participate

Not applicable.

Consent for publication

All authors consent publication.

\section{Competing interests}

The authors declare that they have no competing interests.

\section{Publisher's Note}

Springer Nature remains neutral with regard to jurisdictional claims in published maps and institutional affiliations.

\section{Author details}

${ }^{1}$ Montevideo Clinical and Research Unit, Avda Italia s/n, Hospital de Clinical, 11600 Montevideo, Uruguay. ${ }^{2}$ UNDP/UNFPA/UNICEF/WHO/World Bank Special Programme of Research, Development and Research Training in Human Reproduction, Department of Reproductive Health and Research, World Health Organization, Avenue Appia 20, 1211 Geneva, Switzerland.

Received: 10 January 2017 Accepted: 23 November 2017

Published online: 13 December 2017

\section{References}

1. World Health Organization (WHO). Maternal Mortality. Fact sheet $N^{\circ} 348$ Updated November 2015. http://www.who.int/mediacentre/factsheets/ fs348/en/. Accessed 26 Oct 2016.

2. United Nations. Open working group of the general assembly on sustainable development goals. 2014. http://undocs.org/A/68/970. Accessed 30 July 2016.

3. Kearns AD, Caglia JM, Ten Hoope-Bender P, Langer A. Antenatal and postnatal care: a review of innovative models for improving availability, accessibility, acceptability and quality of services in low-resource settings. BJOG. 2016;123:540-8.

4. Institute of Medicine (US) Committee on Improving Birth Outcomes; Bale JR Stoll BJ, Lucas AO, editors. Improving Birth Outcomes: Meeting the Challenge in the Developing World. Washington (DC): National Academies Press (US); 2003. Available from: https://www.ncbi.nlm.nih.gov/books/ NBK222097/. doi:10.17226/10841

5. Ikoh M, Udo A, Charles A, Charles J. The influence of "stock out" on healthseeking behavior of low income women in Uyo urban, Akwa Ibom state, Nigeria. Int Q Community Health Educ. 2008-2009:29:257-73.

6. Pasquet A, Messou E, Gabillard D, Minga A, Depoulosky A, Deuffic-Burban S, Losina E, Freedberg KA, Danel C, Anglaret X, Yazdanpanah Y. Impact of drug stock-outs on death and retention to care among HIV-infected patients on combination antiretroviral therapy in Abidjan, Côte d'Ivoire. PLoS One. 2010 O; 5: e13414.

7. Wagenaar H, Gimbel S, Hoek R, Pfeiffer J, Michel C, Manuel J, Cuembelo F, Quembo T, Afonso P, Gloyd S, Sherr K. Stock-outs of essential health products in Mozambique-longitudinal analyses from 2011 to 2013. Tropical Med Int Health. 2014;19:791-801.

8. Leung N, Chen A, Yadav P, Gallien J. The impact of inventory management on stock-outs of essential drugs in sub-Saharan Africa: secondary analysis of a field experiment in Zambia. PLoS One. 2016;11:e0156026. 
9. Biza A, Jille-Traas I, Colomar M, Merialdi M, Requejo J, Althabe F, Aleman A, Bergel E, Cafferata ML, Carbonell A, Chavane L, Crahay B, Delvaux T, Geelhoed D, Gülmezoglu AM, Malapende CR, Melo A, Nguyen MH, Bique N, Widmer M, Temmerman M, Betrán AP. Challenges and opportunities for implementing the WHO antenatal care model in Mozambique: a qualitative study. BMC Health Serv Res. 2015 Sep 2;15:200.

10. Greenwood AM, Bradley AK, Byass P, Greenwood BM, Snow RW, Bennett S, Hatib- $N^{\prime}$ Jie AB. Evaluation of a primary health care programme in the Gambia. I. The impact of trained traditional birth attendants on the outcome of pregnancy. J Trop Med Hyg. 1990;93:58-66.

11. Ouma MN, Chemwolo BT, Pastakia S, Christoffersen-Deb A, Washington S. Pilot study of single-use obstetric emergency medical kits to reduce maternal mortality. Int J Gynecol Obstet. 2012;119:49-52.

12. McDougal L, Moteetee MM, Mohai F, Mphale M, Mahanty B, Motaung B, et al. Lesotho's minimum PMTCT package: lessons learned for combating vertical HIV transmission using co-packaged medicines. J Int AIDS Soc. 2012;15:17326.

13. Darmstadt GL, Hassan M, Balsara ZP, Winch PJ, Gipson R, Santosham M. Impact of clean delivery-kit use on newborn umbilical cord and maternal puerperal infections in Egypt. J Health Popul Nutr. 2009;27:746-54.

14. Hundley VA, Avan BI, Braunholtz D, Graham WJ. Are birth kits a good idea? A systematic review of the evidence. Midwifery. 2012;28:204-15.

15. Benova L, Cumming O, Gordon B, Magoma M, Campbell O. Where there is no toilet: water and sanitation environments of domestic and facility births in Tanzania. PLoS One. 2014;9:e106738.

16. Gon G, Restrepo-Méndez MC, Campbell OM, Barros AJ, Woodd S, Benova L, Graham WJ. Who delivers without water? A multi country analysis of water and sanitation in the childbirth environment. PLoS One. 2016;11:e0160572.

17. Moher D, Liberati A, Tetzlaff J, Altman DG, The PRISMA Group. Preferred reporting items for systematic reviews and meta-analyses: the PRISMA statement. PLoS Med. 2009;62:1006-12.

18. Aleman A, Betran AP, Cafferata ML, Colomar M, Tomasso G. Use of kits for maternal health during antenatal care and delivery: a mixed methods systematic review. PROSPERO 2016 CRD42016043145 Available from: http://www.crd.york.ac.uk/PROSPERO/display_record.php?D=CRD42016043145. ID $=$ CRD42016043145.

19. Glujovsky D, Bardach A, García Martí S, Comandé D, Ciapponi A. PRM2 EROS: a new software for early stage of systematic REVIEWS. Value Health. 2011:14:A564.

20. Higgins J, Green S. Cochrane Handbook for Systematic Reviews of Interventions Version 5.1.0 [updated March 2011]. The Cochrane Collaboration 2011. http://training.cochrane.org/handbook/. Accessed 15 December 2016.

21. Von Elm E, Altman DG, Egger M, et al. The strengthening the reporting of observational studies in epidemiology (STROBE) statement: guidelines for reporting observational studies. Lancet. 2007;370:1453-7.

22. Wong WC, Cheung CS, Hart GJ. Development of a quality assessment tool for systematic reviews of observational studies (QATSO) of HIV prevalence in men having sex with men and associated risk behaviours. Emerg Themes Epidemiol. 2008:5:23.

23. Berra S, Elorza-Ricart JM, Estrada M-D, et al. Instrumento para la lectura crítica y la evaluación de estudios epidemiológicos transversales (A tool for the critical appraisal of epidemiological cross-sectional studies). Gac Sanit. 2008;22:492-7.

24. Fowkes FG, Fulton PM. Critical appraisal of published research: introductory guidelines. BMJ. 1991;302:1136-40.

25. Sanderson S, Tatt ID, Higgins JP. Tools for assessing quality and susceptibility to bias in observational studies in epidemiology: a systematic review and annotated bibliography. Int J Epidemiol. 2007;36:666-76.

26. Guyatt G, Oxman AD, Akl EA, et al. GRADE guidelines: 1. Introduction-GRADE evidence profiles and summary of findings tables. J Clin Epidemiol. 2011;64:383-94.

27. Schiffman J, Darmstadt GL, Agarwal S, Baqui AH. Community-based intervention packages for improving Perinatal health in developing countries: a review of the evidence. Semin Perinatol. 2010;34:462-76.

28. Haws RA, Thomas AL, Bhutta ZA, Darmstadt GL. Impact of packaged interventions on neonatal health: a review of the evidence. Health Policy Plan. 2007;22:193-215.

29. Bhutta ZA, Darmstadt GL, Hasan BS, Haws RA. Community-based interventions for improving perinatal and neonatal health outcomes in developing countries: a review of the evidence. Pediatrics. 2005;115:519-617.

30. Jokhio AH, Winter HR, Cheng KK. An intervention involving traditional birth attendants and perinatal and maternal mortality in Pakistan. N Engl I Med. 2005:352:2091-9.
31. Opportunities for Africa's Newborns: Practical data, policy and programmatic support for newborn care in Africa. Joy Lawn and Kate Kerber, eds. PMNCH, Cape Town, 2006. ISBN-13: 978-0-620-37695-2. ISBN-10: 0-620-37695-3.

32. Balsara ZP, Hussein MH, Winch PJ, Gipson R, Santosham M, Darmstadt GL. Impact of clean delivery kit use on clean delivery practices in Beni Suef governorate, Egypt. Journal of Perinatology. 2009; 10:673-679.

33. Calvert J, Steen M. Homeopathic remedies for self-administration during childbirth. Br J Midwifery. 2007;15:159-65.

34. Dickerson T, Crookston B, Simonsen SE, Sheng X, Samen A, Nkoy F. Pregnancy and village outreach Tibet: a descriptive report of a communityand home-based maternal-newborn outreach program in rural Tibet. J. Perinat. Neonatal Nurs. 2010;24:113-27.

35. Garner P, Lai D, Baea M, Edwards K, Heywood P. Avoiding neonatal death: an intervention study of umbilical cord care. J Trop Pediatr. 1994;40:24-8.

36. Hassan $H$, Jokhio AH, Winter $H$, MacArthur C. Safe delivery and newborn care practices in Sindh, Pakistan: a community-based investigation of mothers and health workers. Midwifery. 2012;28:406-11.

37. Raza SA, Avan BI. Disposable clean delivery kits and prevention of neonatal tetanus in the presence of skilled birth attendants. Int J Gynecol Obstet. 2013;120:148-51.

38. Seward N, Prost A, Copas A, Corbin M, Li L, Colbourn T, et al. Using observational data to estimate the effect of hand washing and clean delivery kit use by birth attendants on maternal deaths after home deliveries in rural Bangladesh, India and Nepal. PLoS One. 2015, e0136152;10

39. Vallely LM, Homiehombo P, Walep E, Moses M, Tom M, Kelly-Hanku A, et al. Feasibility and acceptability of clean birth kits containing misoprostol for self-administration to prevent postpartum hemorrhage in rural Papua New Guinea. Int J Gynecol Obstet. 2016;133:301-6.

40. Winani S, Wood S, Coffey P, Chirwa T, Mosha F, Changalucha J. Use of a clean delivery kit and factors associated with cord infection and puerperal sepsis in Mwanza, Tanzania. Midwifery Womens Health. 2007;52:37-43.

41. Mukasa S. Community-based distribution models for rolling out misoprostol for PPH prevention: experience and lessons learned from Uganda and Tanzania. Int J Gynecol Obstet. 2012;119:S227.

42. Tsu V. Nepal Clean Home Delivery Kit: evaluation of the health impact. 2000 https://www.path.org/publications/files/TS_nepal_cdk_eval.pdf. Accessed 15 Dec 2016.

43. Seward N, Osrin D, Li L, Costello A, Pulkki-Brannstrom AM, et al. Association between clean delivery kit use, clean delivery practices, and neonatal survival: pooled analysis of data from three sites in South Asia. PLoS Med. 2012;9:e1001180.

44. Kapoor SK, Reddaiah VP, Lobo J. Control of tetanus neonatorum in a rural area. Indian J Pediatr. 1991;58:341-4.

45. Meegan ME, Conroy RM, Lengeny SO, Renhault K, Nyangole J. Effect on neonatal tetanus mortality after a culturally-based health promotion programme. Lancet. 2001;358:640-1.

46. Quaiyum MA, Prata N, Holston MM, Hossain S, Rahman S. Protecting women who deliver at home from PPH: the use of clean delivery kits in northwestern Bangladesh. Int J Gynecol Obstet. 2012;119:S370.

47. Chavane L, Merialdi M, Betran AP, Requejo-Harris J, Bergel E, Aleman A, Colomar M, Cafferata ML, Carbonell A, Crahay B, Delvaux T, Geelhoed D, Gülmezoglu M, Malapende CR, Melo A, Nguyen MJ, Osman NB, Widmer M, Temmerman M, Althabe F. Implementation of evidencebased antenatal care in Mozambique: a cluster randomized controlled trial: study protocol. BMC Health Services Research. 2014; (14): 228. doi: 10.1186/1472-6963-14-228.

48. Manandhar D, Osrin D, Shrestha B, Mesko N, Morrison J, Tumbahangphe KM, Tamang S, Thapa S, Shrestha D, Thapa B, Shrestha JR, Wade A, Borghi J, Standing H, Manandhar M, Costello AM; Members of the MIRA Makwanpur trial team. Effect of a participatory intervention with women's groups on birth outcomes in Nepal: cluster randomized controlled trial. Lancet. 2004; 364:970-979.

49. Tripathy P, Nair N, Barnett S, Mahapatra R, Borghi J, Rath S, Rath S, Gope R, Mahto D, Sinha R, Lakshminarayana R, Patel V, Papel C, Prost A, Costello A. Effect of a participatory intervention with women's groups on birth outcomes and maternal depression in Jharkhand and Orissa, India: a clusterrandomised controlled trial. Lancet. 2010;375:1182-92.

50. Colomar M, Cafferata ML, Aleman A, Tomasso G, Beltran AP. Supply kits for antenatal and childbirth care: a mixed-methods systematic review, the qualitative approach. Reprod Health. 2017;14:48. 